\title{
DIVISÃO E DISTRIBUIÇÃO DOS NERVOS FRÊNICOS NO DIAFRAGMA DE BÚFALO (Bubalus bubalis LINNAEUS, 1758) DA RAÇA MURRAH
}

\author{
DIVISION ANID DISTRIBUTION OF PHRENIC NERVES IN THE DIAPHRAGMS OF MURRAH BUFFALOES \\ (Bubalus bubalis LINNAEUS, 1758)
}

Armóbio Amanajás 'TOC ANTINS NETO'; Nilson FERREIRA²; Maria Angélica MIGI.JNO; Wilson Machado de SOLZZA'

\begin{abstract}
RESUMO
Com base na dissecação de 30 diafragmas de búfalos. adultos. machos, da raça Murrah para estudar a divisão e a distribuição dos nervos frênicos. os autores chegaram às seguintes conclusōes: os nervos frênicos terminam por bifurcação, tanto à direita $(36,7 \%)$, quanto à esquerda $(100,0 \%)$, ramo dorsal e tronco ventrolateral. Terminam por bifurcação somente à direita $(46,7 \%)$. em ramo ventral e tronco dorsolateral. Terminam por trifurcação, apenas à direita $(16.7 \%)$ em ramos dorsal, lateral e ventral. Dividem-se simetricamente $(40 \%)$ por bifurcação, em ramo dorsal e tronco ventrolateral. O ramo dorsal do nervo frênico esquerdo $(23,3 \%)$ apresenta distribuição heterolateral. O ramo ventral do nervo frênico esquerdo ( $10 \%$ ) mostrou-se com distribuição heterolateral. Conexōes homolaterais foram encontradas entre os ramos dorsal e lateral direitos $(3,3 \%)$ entre filetes dos ramos dorsal esquerdo (3,3\%), ou do lateral esquerdo $(3.3 \%)$.
\end{abstract}

UNITERMOS: Búfalos: Diafragma; Nervo Frênico

\section{INTRODUÇĀO E IITTERATLRA}

O búfalo da Índia, um dos maiores bois selvagens do mundo. vive em pastos altos, às margens de florestas no Nepal, Terai e nas planicies de Ganges e Brahmaputra.

De acordo com informaçōes da Associação Brasileira de Criadores de Búfalos, somente os búfalos indianos ou asiáticos foram domesticados, e estes podem ser divididos em dois tipos básicos, por certos caracteres anatômicos e requisitos ecológicos.

Oprimeiro grupo compreende o búfalo de rio ("water buffalo") encontrado na Índia, no Paquistão, no Egito c na região mediterrânea. O segundo grupo ("swamp buffalo") inclui os animais de pântano do Sudoeste Asiático.

No Brasil existem os dois tipos, ou seja, o búfalo indiano (Murrah, Jaffarabadi e Mediterrâneo) e o búfalo de pântano (Carabao ou Rosilho).

Os búfalos Murrah são animais considerados bons produtores de carne e de leite e, dentre as raças exploradas no Brasil, merecem destaque não somente por suas aptidões como também pelas facilidades de manejo, tratando-se de animais dóceis.
Diante da quantidade de animais desta raça que hoje compōem o rebanho brasileiro, e interessados em dados para o estudo da anatomia do búfalo, realizamos este trabalho, cujo objetivo visa particularidades relativas ao músculo diafragma e sua inervação.

Na revisão de literatura pertinente ao assunto os autores de tratados especializados em anatomia (ZIMMERL ${ }^{19}$ s.d.; LESBRE ${ }^{10}$, 1923; ELLENBERGER; BAUM" ${ }^{\circ}$ 1932; BRUNI; ZIMMERL ${ }^{3}, 1947$; GONZALES y GARCIA; GONZALEZ ALVAREZ", 1961; DOBBERSTEIN; HOFFMAN ${ }^{5}$, 1964; SISSON; GROSSMAN ${ }^{17}$, 1963; SCHWARZE; SCHRODER '", 1972; GETTY'. 1975) fazem menção somente à espécie equiina e relatam apenas que os nervos frênicos chegam ao centro tendíneo e dirigem-se, após sucessivas divisões, à parte muscular do diafragma.

Trabalhos específicos sobre o assunto realizados, inicialmente, por BERTELLI ${ }^{1.2}$ (1894, 1933), foram feitos em grandes ruminantes (Bos taurus), por PANCRAZI ${ }^{14 / 5}$ (1926, 1941), GIROLLA $^{k}$ (1955); (Bos indicus) por NEVES ${ }^{12}$ (1968), SOUZA et al. ${ }^{18}$ (1984) e em (Bubalus bubalis) por MIGLINO) et al." (1985).

- Professor Adjunto - Faculdade de Medicina Veterinária da Universidade Federal do Pará

2. Professor Associado - Faculdade de Medicina Veterinária é Zextecnia da USP

3 - Professor Adjunto - Faculdade de Medicina Veterinária da UNI:SP - "campus" de Araçatuba 
TOCANTINS NE:TO. A. A.: IF:RRI:IRA. N.: MICil.INO. M. A.: SOUZ.A. W. M. Divisūo è disıribuição dos nervos frenicos no dialragma de búfalo (Bubalus bubalis l.innacus, 1758) da raça Murrah. Brał. J. vet. Res. anim. Sci., São Paulo, v. 31, n. I, p. 1-7, 1994.

\section{MATERIAL, E MÉTODO}

Utilizamos 30 diafragmas de búfalos da raça Murrah, adultos, machos, provenientes dos Estados de São Paulo, Mato Grosso do Sul e Pará.

As peças foram reliradas dos animais nos frigoríficos mediante seç̧ão dos nervos frênicos, incisão da parte muscular c periférica ao longo das suas inserções costal, esternal c lombar e separação das estruluras vizinhas. mantendo em posição o esôfago, nervo vago, a aorla c veia cava caudal.

Os diafragmas foram fixados distendidos sobre uma base rígida em solução aquosa de formol a $10 \%$ por 72 horas.

De acordocom a NOMINA ANATOMICA VETERINARIA ${ }^{13}$ (1983) identificamos no diafragma uma pars costalis (parto costal), uma pars esternalis (parte esternal) e uma pars lumbaris (parte lombar), correlacionadas por ordem com as costelas, processo xifóide, corpos das quatro ou cinco primeiras vértebras lombares (crus dextrum, pilar direito) e corpos das duas primeiras vértebras lombares (crus sinistrum. pilar esquerdo) além dos folíolos dorsais e ventral do centrum tendineum (centro tendíneo).
A dissecação foi realizada sob lupa estereoscópica Ramsor modelo LI-20 (10 X), e de cada preparação foram tomadas fotografias e feitos esquemas representativos das disposições encontradas.

A nomenclatura utilizada foi a mesma empregada por BERTELL1.1.2(1894, 1933), com as modificações introduzidas por CONDE ${ }^{\dagger}$ (1957).

\section{RESUI,TADOS}

\section{A) Divisão dos Nervos Frênicos}

()s nervos frênicos do búfalo Murrah bifurcam-se pouco antes de atingirem o difragma em ramo dorsal e tronco ventrolateral, $36,7 \%$ dos casos à direita e em todas as preparações à esquerda. A bifurcação pode dar-se em ramo ventral e tronco dorsolateral, $46,7 \%$ à direita. Também somente à direita verificou-se em 16,7\% dos casos a trifurcação em ramos dorsal, lateral e ventral.

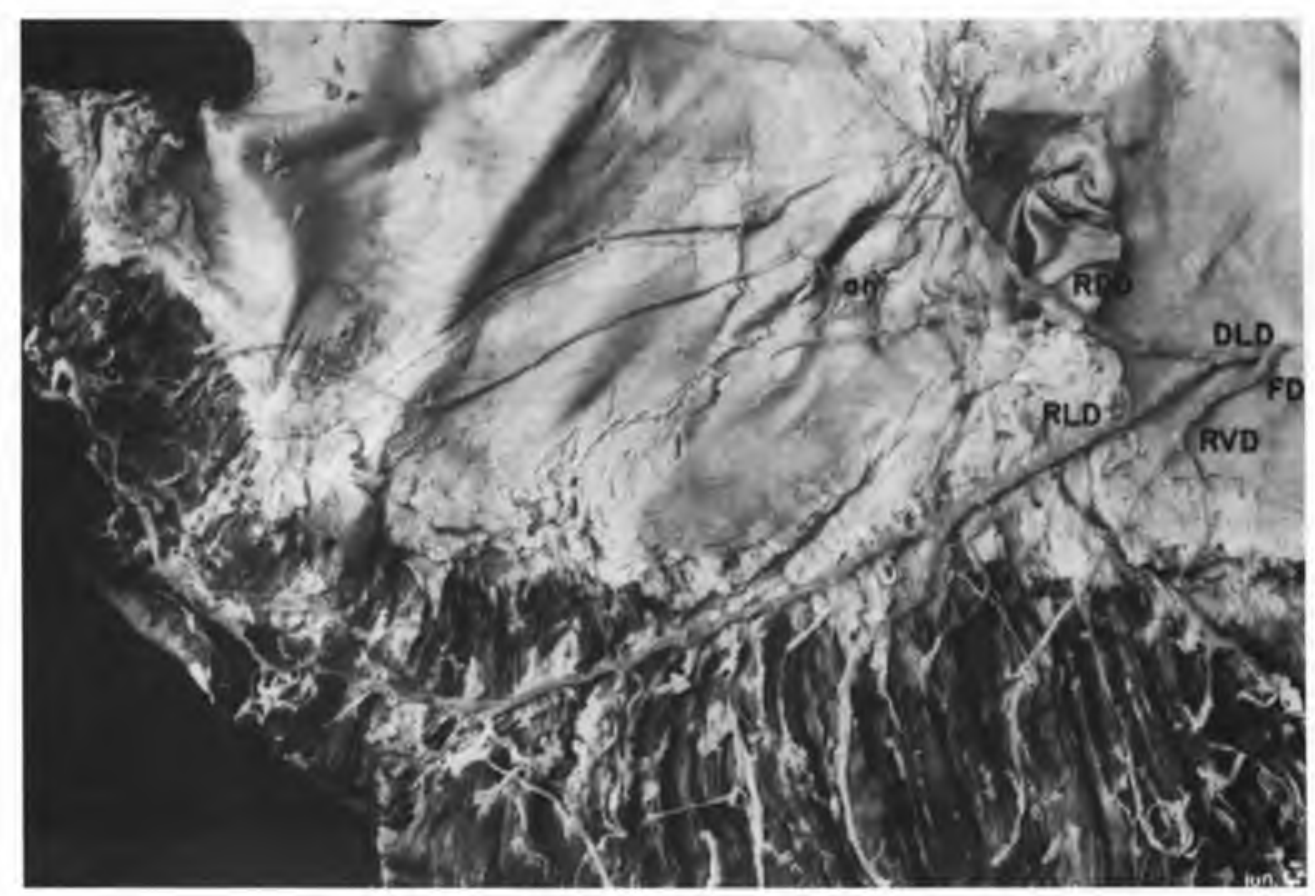

FIGURA 1

Fotografia do dialragma de búfalo da raça Murrah. macho, adulto, visto pela face torácica. O nervo frénico direito (FD) divide-se en ramo ventral (RVD) e tronco dorsolateral (I)I D). Os ramos dorsal (RI)D) e lateral (RI.D) direitos unem-se por meio de um filcie anastomótico (an). Reduçāo $8 \mathrm{X}$. 
TOCANTINS NETO, A. A.; \&ERREIRA. N.; MIGLINO, M. A.; SOUZA, W. M. Divisāo e distribuição dos nervos frênicos no diafragma de búfalo (Bubalus bubalis Linnaeus, 1758) da raçal Murrah. Braz. J. vet. Kes. anim. Sci., Säo P’aulo, v. 31, n. I, p. 1-7. 1994.



FIGURA 2

Dialraggma de búfalo da raça Murrah, macho, adulto, visto pela face torácica. Os nervos frểnico direito (FD) e esquerdo (FE) bifurcam-se simetricamente em ramo dorsal (RDD-RIDE) e tronco ventrolateral (VLD-VLE). Hstes. por sua vě. enderę̧am-se. respectivamente às partes lombares. esternais e costajs (regiāo ventrolateral) correspondentes. O ramo dorsal esçuerdo (RI)F) emite um lílete nervoso (seta) destinado ao pilar direito. Redução 8X.

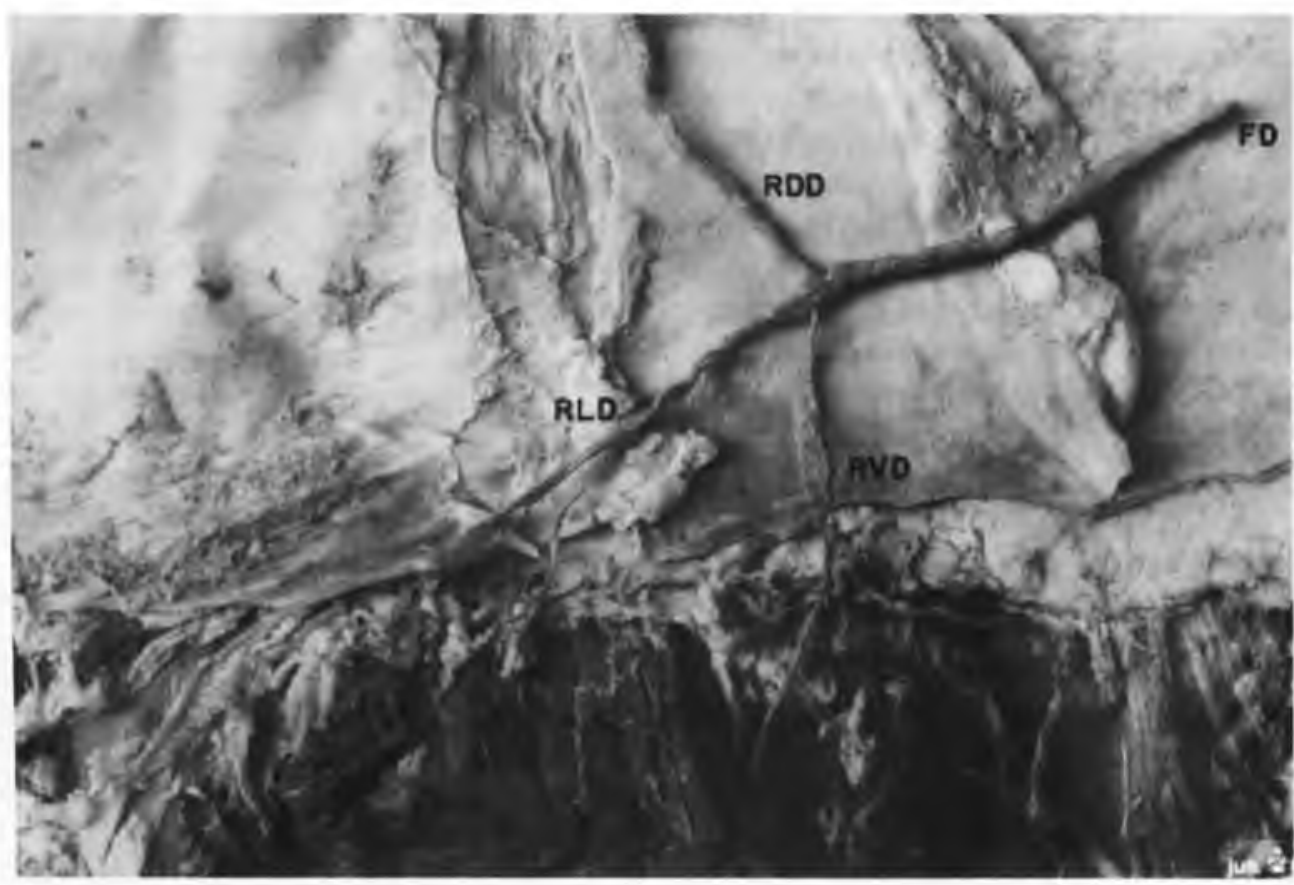

FIGURA 3

Diafragma de búfalo da raça Murrah, macho, adulto, visto pela face torácica, apresentando o nervo frênico direito) (FI)) trifurcando-se em ramos dorsal (RDD), lateral (RI.D) e ventral (RVD). Reduçio 8X. 


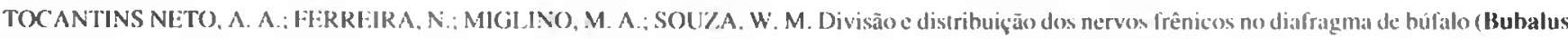
buhalis I innateus. 1758) da raça Murralı. Bra\%. J. vet. Res. anim. Sci., Sāo Pauulo, v. 31, n. I, p. I-7, 1994.



FIGURA 4

Diafragma de búfalo da raça Murrah, macho, adulto, visto pela face torácica. O nervo frênico esquerdo (FE) bifurcase num ramo dorsal e num troneo ventrolateral. Do ramo dorsal esquerdo (RDE) emerge um lilete. que inerva a parte lombar opostal (scta).

\section{B) Distribuição dos Nervos Frênicos}

Os ramos dorsais dos nervos frênicos distribuem-se somente na parte lombar homolateral em 73,3\% à direita e em 76,7\% das preparaçōes à esquerda. Particularmente o ramo dorsal direito dá origem, em $20 \%$ das peças, a um pequeno filete que se dirige à parede da veia cava caudal.

Em uma única ocasião, o ramo dorsal direito, além de inervar a parte lombar direita, emite dois filletes nervosos os quais destinam-se à parte costal (região dorsolateral) homolateral. Este mesmo arranjo é notado em outra peça na qual o ramo dorsal inerva a parte lombar direita e a veia cava caudal e envia um ramo à parte costal.

Por sua vez, o ramo dorsal esquerdo, além de inervar a parte lombar esquerda, emite em 23,3\% dos casos um ramúsculo endereçado à parte lombar direital e que em seu percurso cruza ventralmente o hiato esofágico.

Os ramos laterais e ventrolaterais da parte costal encontramse em todas as dissecações à esquerda, e em 93.3\% das peças à direita. Em dois casos isolados, o ramo lateral direito, além, de inervar as regióes correspondentes, emite um $(3,3 \%)$ ou dois $(3,3 \%)$ filetes para o folíolo dorsal direito. Os ramos ventrais distribuem-se por sua vez na região ventrolateral da parte costal e parte esternal correspondente, $90 \%$ à direita e
$86,7 \%$ à esquerda. Em $6,6 \%$ das preparaçñes à direita, o ramo ventral destina-se somente à porção ventrolateral da parte costal. Ainda, à direita. ele incrva além da parte correspondente, of folíolo ventral $(3,3 \%)$.

À esquerda vimos o ramo ventral alcançando em $10 \%$ dos casos, além da parte correspondente, a parte estemal oposta. Num caso isolado $(3,3 \%)$ o ramo ventral esquerdo inerva as partes correspondentes. parte esternal oposta e folíolo ventral.

\section{C) Conexões dos ramos derivados dos nervos frênicos}

As conexões eneontradas entre liletes derivados dos nervos frênicos foran sempre homolaterais c ocorreram em apénas três ocasiōes. A primeira delas $(3,3 \%)$ foi verificada entre filetes derivados dos ramos dorsal e lateral direito. A segunda $(3,3 \%)$ entre filetes derivados do ramo lateral esquerdo, na região dorsolateral da parte costal. Finalmente em uma única peça $(3,3 \%)$ foi possível verificar uma conexão entre filetes derivados do ramo dorsal esquerdo.

\section{COMENTÁRIOS}

Apenas MIGI .INOet al. " ( 1985 ) referem-se a búfalos Murrah. pois as pesquisas relativas aos demais ruminantes somente apresentam dados atinentes à comparação entre Bos taurus e Bos indicus. 
Os compêndios de Anatomia Veterinária (ZIMMERL." ,s.d.; LESBRE ${ }^{10}, 1923$; ELLENBERGER; BAUM",1932; BRUNI; ZIMMERL ${ }^{3}, 1947$; GONZALIZ y GARCIA; GONZALEZ ALVAREZ',1961; DOBBERSTIIN: HOFFMAN", 1964; SISSON; GROSSMAN ${ }^{17}, 1963$; SCHWARZE; SCHRODER ${ }^{16}$, 1972 e GETTY ${ }^{7}$, 1975), ao tratarem especificamente do assunto, referem-se somente ao eqüino, registrando para outras espécies domésticas apenas as diferenças de maior realce, tais como a afirmação de que os nervos após sucessivas divisões dirigem-se à parte muscular do diafragma. Este fato, a nosso ver, torna o confronto dos nossos resultados com tais relatos, pouco viável.

No tocante à divisão dos nervos frênicos em buifalos, assinalamos 3 ramos principais cognominados, segundo BERTELLI ${ }^{2}$ (1933), dorsal, lateral e ventral. Esta afirmação soma-se àquelas descritas por outros autores (PANCRAZI ${ }^{14.15} 1926$. 1941; GIROLLA ${ }^{8}, 1955$; NEVES ${ }^{12}, 1968$; SOUZA at al. ${ }^{18}$, 1984; MIGLINO et al. ", 1985) us quais descrevem os troncos dorsolateral e ventrolateral, considerando os três ramos frênicos, que se dirigem às partes correspondentes do diafragma.

Quanto ao nervo frênico direito, há maior freqüência $(46,7 \%)$ de um ramo ventral e um tronco dorsolateral, disposição também encontrada por SOUZA el al. ${ }^{1 \times}(1984)(16,6 \%)$ e por MIGLINO et al." ( 198.5$)(53,3 \%)$. De outra parte, vimo-lo dividir-se em um ramo dorsal e tronco ventrolateral $(36,7 \%)$, observação que aparece como a mais frequente nas pesquisas de PANCRAZI ${ }^{14.15}$ (1926. 1941); GIROLLA* (1955) e NEVES $^{12}$ ( 1968$)(90 \%)$; SOU $/ A$ et al. ${ }^{1 \%}$ ( 1984$)(60 \%)$ e, menos freqüentemente naquela desenvolvida por MIGLINO et al." (1985) (26.6\%) realizada nos búfalos Jaffarabadi. Relativamente à modalidade de divisão por trifurcação, apreciatmo-la em algumas preparações (16,7\%), tal como NE.VES ${ }^{12}$ (1968) (10\%): SOUZA et al. ${ }^{18}(1984)(23.3 \%)$ e, por fim. por MIGLINO et al. " ( 1985 ) (20\%), este último trabalho referente a búfalos Jaffarabadi.

Relativamente à divisão terminal do nervo frênico esquerdo, notamos, em todos os casos, a presença de um ramo dorsal e um tronco ventrolateral, semelhante à encontrada por PANCRAZ. ${ }^{14.15}(1926,1941)$ para os bovinos, por GIROLI.A ${ }^{8}$ (1955) e por NEVES ${ }^{12}(1968)(83,3 \%$ e $85 \%)$, por SOUZA et al. ${ }^{18}$ (1984) (60\%), e que se aproxima dos dados encontrados por MIGLINO et al." (1985) (86.6\%) no concernente aos bubalinos.

Analisando agora a distribuição dos nervos frênicos no diafragma, verificamos como PANCRAZI $I^{1+15}$ (1926. 1941) (em todos os casos), GIROLLA* (1955) (em quase todos os casos), NEVES ${ }^{12}$ ( 1968$)(96,6 \%$ à direita e $20 \%$ à esquerda), SOUZA et al. ${ }^{18}$ ( 1984$)(97 \%$ à direita e $26.7 \%$ à esquerda) e MIGLINO et al. " (1985) (26,6\% à direita e $6,6 \%$ à esquerda) o ramo dorsal inervando somente a parte lombar correspondente (73,3\% à direita e $76,7 \%$ à esquerda). Em outras ocasiões, pudemos observar filetes derivados do ramo dorsal direito endereçados à veia cava caudal (20\%), fato que no búfalo Jaffarabadi ocorreu em 40\% dos preparados e nos bovinos da raça Nelore em apenas um caso (3\%). Uma das peças por nós dissecadas $(3,3 \%)$ mostrou que do ramo dorsal direito originavam-se filetes não somente destinados à veia cava caudal como também ao folíolo dorsal direito, assim como pudemos observar, num outro diafragma, filcte derivado do cilado ramo e dirigido à região dorsolateral da parte costal homóloga, ocorrência apontada por NEVES ${ }^{12}$ (1968) e por GIROLLA ${ }^{k}$ (1955) $\mathrm{cm}$ igual percentagem $(3,3 \%)$.

À esquerda observamos que o ramo dorsal inervava, no búfalo Murrah, além da parte lombar homóloga, a parte lombar oposta $(23,3 \%)$, mediante filete que percorria ventralmente o hiato esofágico, fato que vem confirmar o que foi observado no búfalo Jaffarabadi no mesmo total de diafragmas e que é citado por PANCRA $I^{1415}$ (1926, 1941); GIROLLA ${ }^{8}$ ( 1955); NEVES $^{12}$ (1968) (80\%) e por SOUZA ct al. ${ }^{18}(1984)(73,3 \%)$ nos bovinos.

Os ramos laterais inervam, conforme afirmam os autores especializados e de acordo com nossos resultados, as partes costais homolaterais. Convém lembrar que NEVES ${ }^{12}$ (1968); SOUZA et al. ${ }^{18}$ (1984) e MIGLINO et al. " ( 1985 ), analisando o referido ramo, verificaram que ele se distribui exclusivamente à parte costal em 93,3\% das dissecações à direita, do mesmo modo que pudemos também observá-lo distribuindose no folíolo dorsal direito $(6,7 \%)$, como MIGLINO et al." (1985) $(6.6 \%)$

À esquerda consignamo-lo em todas as oportunidades, orientando somente à parte costal homolateral. Embora os outros autores tenham apontado modalidades diversas de distribuição deste ramo, constatamos que quase todos especificam nas suas descrições que este arranjo configura a maioria dos casos.

Prosseguindo no exame comparativo, agora relativamente aos ramos ventrais, verificamos que o ramo ventral direito distribui-se na porção ventrolateral da parte costal (sempre) e na parte esternal (93,3\%) das peças, resultado muito próximo dos de PANCRAZI ${ }^{14.15}$ (1926, 1941); $\operatorname{GIROLLA}^{*}$ (1955); NEVES ${ }^{12}$ (1968); SOUZA et al. ${ }^{18}$ (1984) e MIGLINO et al. ${ }^{11}$ (1985). Com maior freqüência deparamo-nos à direita a exemplo de NEVES ${ }^{12}$ ( 1968$)(76.6 \%)$; SOUZA et al. ${ }^{18}$ ( 1984$)$ (em todos os casos) MIGLINO et al." (1985) (86,6\%), com o ramo ventral alcançando somente as partes costal e esternal correspondentes $(93,3 \%)$. Eventualmente no búlalo Murrah, o ramo ventral direito dirige-se à parte costal heterolateral, o 
TOCANTINS NETO. A. A.; IF:RREIRA. N.; MIGI.INO. M. A.; SOU\%.A, W. M. Divisão c distribuição dos nervos frênicos no diafragıma de búfalo (Bubalus bubalis Linnacus, 1758) da raça Murrah. Braz. J. vet. Res. anim. Sei., São Paulo, v. 31, n. I. p. 1-7. 1994.

mesmo acontecendo com os animais estudados por MIGLINO et al. "' (1985) (6,6\%). Saliente-se, todavia, o arranjo incomum encontrado somente no nosso material, no qual o aludido ramo distribui-se na região ventrolateral da parte costal direita e no folíolo ventral $(3,3 \%)$.

No tocante à distribuição do ramo ventral esquerdo, achamolo mais frequientemente $(86,7 \%)$ como aponta GIROLLA ${ }^{8}$ (1955), a distribuir-se às porções ventrolateral das partes costal e esternal esquerdas. Porém, em alguns casos (10\%), pode enviar ramúsculo à parte esternal direita, ou ao folíolo ventral $(3,3 \%)$. Esta observação é semelhante às de PANCRAZI ${ }^{14}$ is $(1926.1941)$ e NEVES ${ }^{12}$ (1968), os quais encontraram o citado ramo a distribuir-se na porção ventral da parte costal e esternal esquerda $(86,6 \%)$ e, a emitir um filete que, tendo origem no ramo ventral, atravessa o folíolo ventral e dirige-se à região estemal oposta $(13,3 \%)$.

Por fim, identificamos nos búfalos Murrah distribuição preponderantemente homolateral dos nervos frênicos direito e esquerdo, fato que a nosso ver sugere que em possiveis estudos experimentais, a seç̧ão de um deles acarretaria degeneração da metade correspondente do diafragma.

Considerando as conexões verificadas entre filetes derivados dos nervos frênicos direito c esquerdo, encontramo-las sempre homolaterais, e este resultado difere daqueles descritos por GIROLLA ${ }^{8}$ (1955), quando da união de ramúsculos derivados dos dois ramos ventrais. Vale dizer que as modalidades de conexões apresentadas pelos búfalos da raça Murrah foram igualmente registradas nos búfalos da raça Jaffarabadi e nos bovinos.

\section{CONCLUSÕES}

1) Os nervos frênicos terminam à direita $(36,7 \%)$ e à esquerda $(100 \%)$ em ramo dorsal e tronco ventrolateral;
2) Os nervos frênicos terminam à direita $(46,7 \%) \mathrm{cm}$ ramo ventral e tronco dorsolateral;

3) Os nervos frênicos terminam à direita $(16,7 \%)$ trifurcandose em ramos dorsal, lateral e ventral;

4) Os nervos frênicos terminam, em cada lado, (36,7\%) em ramo dorsal e tronco ventrolateral;

5) Os nervos frênicos, em conjunto, terminam à direita (16,7\%) trifurcando-se cm ramos dorsal, lateral e ventral e. à esquerda $(16,7 \%)$, bifurcando-se em ramo dorsal e tronco ventrolateral;

7) Os ramos dorsais dos nervos frênicos inervam à direita $(73,3 \%)$ e à esquerda $(76,7 \%)$ a parte lombar homolateral; nos outros casos, à direitra $(26,6 \%)$ e à esquerda $(23,3 \%)$. Distribuem-se também a outras estruturas, tais como: à direita (20\%), à veia cava caudal; à esquerda $(23,3 \%)$ à pequena região da parte lombar direita; à dircita $(3,3 \%)$ à região dorsolateral da parte costal do mesmo lado $e$, à direita $(3,3 \%)$ à veia cava caudal e ao folíolo dorsal direito;

8) Os ramos laterais dos nervos frênicos inervam à direita $(93,3 \%)$ e à esquerda $(100 \%)$ as porções dorsolateral e ventrolateral da parte costal;

9) Os ramos ventrais dos nervos frênicos dirigem-se à direita $(90 \%)$ e à esquerda $(86.7 \%)$ à porção ventrolateral da parte costal e parte esternal do mesmo lado.

10) Existem conexões ou "anastomoses" nervosas homolaterais à direita $(3,3 \%)$ entre o ramo dorsal direito e o lateral do mesmo lado, além de outra, entre o referido ramo anastomótico e um filete derivado do ramo dorsal que se distribui na região dorsolateral da parte costal.

\section{SUMMARY}

Based on the dissection of 30 diaphragms of adult male buffaloes of the Murrah breed, the authors studied the division and distribution of the phrenic nerves and the results found were as the follow: the phrenic nerves terminate in bifurcation both in dorsal branches and ventrolateral trunks at right $(36.7 \%)$ and into left (100\%) sides. The phrenic nerves terminate in bifurcation only to the right $(46.7 \%$ ) branches and dorsolateral trunks. The phrenic nerves terminate by trifurcation, only at the right sides ( $16.7 \%)$, in dorsal, lateral and ventral branches. The phrenic nerves were divided symmetrically $(40 \%)$ by bifurcation into dorsal branches and ventrolateral trunks. The dorsal branches of the left phrenic nerves $(23.3 \%$ ) had heterolateral distribution. The ventral branches of the left phrenic nerve $(10 \%)$ had heterolateral distribution. Homolateral connections were verified between the dorsal and lateral right branches (3.3\%); between divisions of the left dorsal branches $(3.3 \%)$ or left lateral branches $(3.3 \%)$.

UNITERMS: Buffaloes; Diaphragm: Phrenic nerve 
TOCANTINS NETO, A. A.; FERREIRA, N.; MIGLINO, M. A.; SOUZA. W. M. Divisão e distribuiçāo dos nervos frênicos no diafragma de búfalo (Bubalus bubalis L.innaeus, 1758) da raça Murrah. Braz. J. vet. Res. anim. Sci., São Paulo, v. 31, n. 1, p. I-7, 1994.

\section{REFERÊNCIAS BIBLIOGRÁFICAS}

01-BERTELLI, D. Contributo alla anatomia del diaframma nei carnivori. Mon. Zool. Ital., v.1, n.10, p. $211-5$, 1894

02-BERTELLI, D. Distribuzione dei nervi frenici nel - diaframma dei mammiferi.Arch. Ital. Anat. Embriol., v.32, n.l, p.110-4, 1933

03-BRUNI, A.C.; ZIMMERL, U. Anatomia degli animali domestici. 2.ed: Milano, Francesco Vallardi, 1947.

(14-CONDE, R. Estudo anatômico sobre a distribuição dos nervos frênicos no músculo diafragma de Canis familiaris. Arq. Esc. Sup. Vet. Univ. Rural Est. M.Gerais, v.10, p.329-65, 1957.

05-DOBBERSTEIN, J.; HOFFMAN, G. Lehrbuch der vergleichendem Anatomie der Haustiere. Leipzig, S. Hirzel, 1964. p.165-7.

06-EllenberGer, W.; BAUM, H. Handbuch der vergleichender Anatomie der Haustiere. Berlin, J. Springer, 1932.

07-GETTY, R. Sisson and Grossman's The anatomy of the domestic animals. 5.ed. Philadelphia, W.B. Saunders, 1975. v.1, p.1127.

08-GIROLLA, W. Der Nervus phrenicus des Rindes. Seinc Morphologic, Topik, Innervations-Und Funktionaufgaben. Wien, 1955. (Inaugural dissertation) - Tierarztlichen Hotchsculhe.

09-GONZALEZ, y GARCIA, J.; GONZALEZ ALVAREZ, $R$.Anatomia comparada de los animales domesticos. Madrid, Gráfica Canales, 1961.

10-LESBRE, F.X.Précis d'anatomie comparée des animaux domestiques. Paris, J.B. Baillière, 1923 p.668-9.
11-MIGLINO,M.A.; PRADA, 1.S.;SOUZA, W.M. Ramificação e distribuição dos nervos frênicos no diafragma em búfalo da raça Jaffarabadi.Rev. Fac. Med. Vet.Zootec. Univ. S.Paulo, v.22, n.2, p.97-106, 1985.

12-NEVES, I.P. Estudo anatômico da distribuição dos nervos frênicos no músculo diafragma de bovinos (fetos). Veterinária, Rio de Janeiro, v.21, p.30-45, 1968.

13-NOMINA ANATOMICA VETERINARIA, revised by the International Committee on Veterinary Gross Anatomical Nomenclature. 3.ed. Ithaca, World Association of Veterinary Anatomists, 1983

14-PANCRAZI, G. Sulla distribuzione del nervo frenico nel diaframma dei mammiferi. (Nota preventiva) Atti $R$. Istit. Veneto Sci. Let. Arti., v.85, n.2, p.345-7, 1926.

15-PANCRAZI, G. Ricerche intorno alla distribuzione del nervo frenico nel diaframma dei mammiferi. Arch. Zool. Ital., v.29, p.413-50, 1941.

16-SCHWARZE, E.; SCHRODER, L. Compendio de anatomia veterinária. Zaragoza, Acribia, 1972.

17-SISSON, S.; GROSSMAN, J.D.Anatomia de los animales domésticos. Barcelona, Salvat, 1963.

18-SOUZA,W.M.;PEREIRA,J.G.L.; ALBUQUERQUE,J.F.G Contribution to the study of diaphragm innervation in Nelore bovines. Anat. Anz., v. 155, p. 317-23, 1984.

19-ZIMMERL, U. Sistema nervoso. In: BOSSI, V.; CARADONNA, G.B.; SPAMPANIO, G.; VARALDI, U.; ZIMMERL. U. Trattato di anatomia veterinaria. Milano, Francesco Vallardi, s.d. v.3, p.228-9. 